\title{
Giant Pulmonary Echinococcus Cyst
}

\author{
Christos Simoglou ${ }^{1}$, Dimitrios Gymnopoulos ${ }^{2}$
}

'Department of Cardiothoracic Surgery, University General Hospital of Evros, Greece ${ }^{2}$ Private Clinic, Komotini, Greece

\section{ABSTRACT}

This is a case report of a 67 year-old male farmer and heavy smoker with 3 packs of cigarettes, who presented with 12 day history of fever and haemoptysis. On chest X-ray a mass was observed at the right lower lobe right lung. He underwent full clinical, laboratory, and endoscopic evaluation. No enlargement of lymph nodes was observed on the CT and also highlighted giant mass in the right lower lobe, of the right lung. The patient underwent surgery which revealed a large viable hydatid cyst measuring $10,3 \times 8,5 \times 7,3 \mathrm{~cm}$, with irregular margins and mixed (solid and cystic) content.

Key words: Echinococcus granulosus, pulmonary echinococcosis, thoracotomy, pulmonary cysts

\section{INTRODUCTION}

Hydatid disease is caused by infection with a small tapeworm parasite called by Echinococcus granulosus, is the most frequently encountered type of anthropozoonosis, and it has been known for centuries. Due to the traditional close association between sheep, dogs and humans, Echinococcus granulosus is endemic in many parts of the word and particularly the Mediterranean countries including Greece (1). Humans are exposed less frequently to E. multilocularis, which causes alveolar echinococcosis. Although it can involve almost every organ of the body, the lung is the second site in frequency, following hepatic infestation, in adults and is the predominant site for hydatidosis in children. The lung facilitates the growth of the cyst due to its compressible nature and the presence of negative pressure. Clinically, a small hydatid cyst in the lung often causes no problems and may remain asymptomatic, but a large cyst may cause pressure symptoms such as chest pain, cough, dyspnea and hemoptysis, as well as allergic reactions including anaphylaxis. Rupture of a pulmonary hydatid cyst into a neighboring or more remote cavity, either spontaneous or iatrogenic is termed a "complicated cyst" and is associated with higher postoperative morbidity and mortality (2). Classically, the chest X-ray shows an intact cyst as a sharply demarcated round to oval

\section{Corresponding author:}

Christos Simoglou, MD

Department of Cardiothoracic Surgery,

University General Hospital of Evros, Greece

E-mail: simoglouchristosdoctor@yahoo.com
Received: 11.09 .2016

Accepted: 21.11.2016

Copyright (๑) Celsius Publishing House 
homogenous mass of variable size. Computed tomography (CT) offers high sensitivity and specificity for the definitive diagnosis of the disease.

\section{CASE REPORT}

A 67 year-old man, farmer, was transferred to the chest hospital for further evaluation of a lung mass revealed on the chest X-ray, which is complicated with fever and haemoptysis. The patient had presented to the local general practitioner 12 days earlier complaining of a few episodes of minor hemoptysis. The physician prescribed an oral antibiotic, but when the hemoptysis, chest X-ray and computed tomography (CT) he performed, revealed a mass in close contact with the hilum of the right lung. The mass had a maximum diameter 9,3 $37,5 \times 6,3 \mathrm{~cm}$, irregular margins and a mixed solid and cystic content. Lymph nodes appeared non-enlarged (or normal) on chest CT (figures 1 and 2). The patient was admitted to the provincial hospital four days from the beginning of the symptoms, with fever and continued hemoptysis, from where he was transferred after five days to the chest hospital for further evaluation and management.

The patient was a smoker and did not report any weight loss. He had a history of hypertension and coronary artery disease. His maximum temperature did not exceed $38,2^{\circ} \mathrm{C}$. The heart sounds were dual without audible murmurs; the blood pressure was 140/90 mmhg and the pulse rate $80 / \mathrm{min}$. There were no abdominal or neurological findings and no edema or palpable lymph nodes. Ear, nose and throat examination was negative for bleeding.

CT scan of the brain and upper abdomen showed no abnormal findings. The bronchoscopy revealed gross bleeding and a thickening of the mucosa at the entrance of the medial basal bronchus of the right bronchial tree. The rest of the upper lower respiratory tract was normal. Brushings were taken from the suspicious area and bronchial secretions were sent for cytology. All work up was negative for malignancy, and only inflammatory cells were demonstrated. In the present study, anti echinococcus antibodies were investigated by ELISA in patient's serum samples, which were routinely delivered to the Medical Microbiology Laboratory of University Faculty of Medicine due to suspected hydatid cyst.

Larger cysts usually need surgical intervention in addition to albendazole (either preoperatively or pre-and postoperatively). The patient underwent

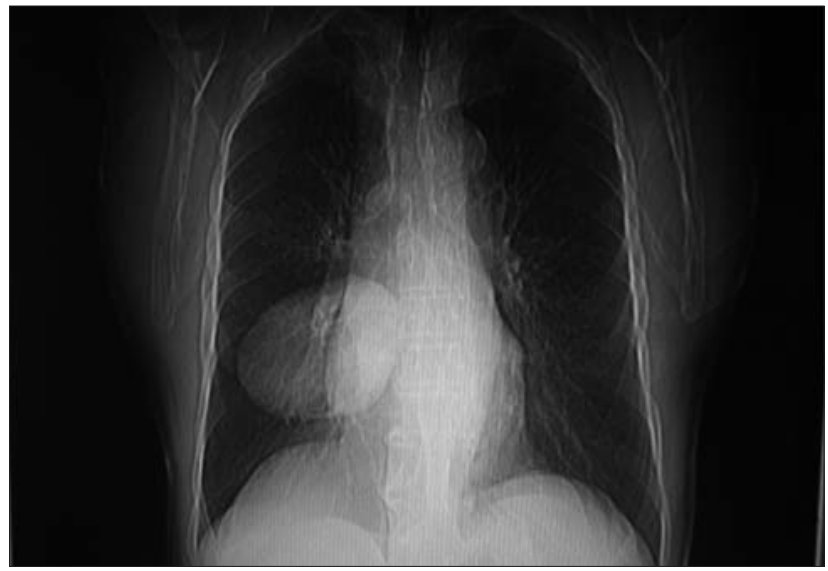

Figure 1 - Chest X-ray showing cyst of the right lung

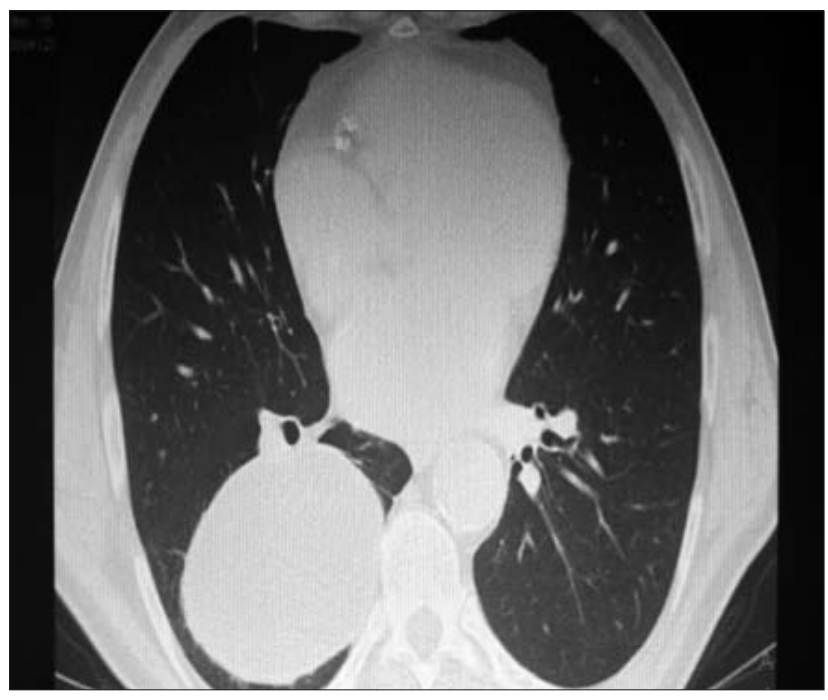

Figure 2 - CT scan of the chest showing a cyst of the right lung

exploratory thoracotomy ten days after admission. Following right posterolateral thoracotomy and lysis of adhesions, a right lower lobectomy, including the mass, was performed. Frozen section was negative for malignancy and following lymph node dissection one chest tube was inserted and the chest was closed. In the final histopathological report the diagnosis of a ruptured Echinococcus cyst with small surrounding abscesses was established. All lymph nodes were negative for malignancy, showing only chronic reactive inflammatory changes. The postoperative period was uneventful and the patient was discharged on the ninth postoperative day. With the establishment of the diagnosis treatment with oral albendazole was given for three months. At follow up 10 months after surgery the patient was doing well without any signs or persistent or recurrent disease. 


\section{DISCUSSION}

The liver is the most common site of cyst formation, followed by the lung, which accounts for $10-30 \%$ of cases, and various other sites in $10 \%$ of cases. In children the lung may be the commonest site of cyst and about $20-40 \%$ also has liver cysts. Pulmonary hydatid disease affects the right lung in almost $60 \%$ of cases. The patient presented here had a solitary lesion in the right lung (3).

Most intact lung cysts are discovered incidentally on chest X-ray. Occasionally an unruptured cyst results in cough, hemoptysis and/or chest pain, hemorrhage or airflow limitation. The commonest complication of symptomatic hydatid pulmonary disease is cyst rupture. The cyst may rupture spontaneously or as a result of trauma or secondary infection. Cyst rupture may be associated with the sudden onset of cough and fever. If the contents of the cyst are expelled into the airway, expectoration of a clear salty or peppery tasting fluid containing fragments of hydatid membrane and scoloces may occur. The patient bronchoscopy was performed which showed no specific findings.

Generalized symptoms of hydatid disease can result from the release of antigenic material and secondary immunological reactions that develop following cyst rupture. Fever and acute hypersensitivity reactions range from urticaria and wheezing to death. Eosinophils are the principal manifestations. Although allergic episodes may develop quite commonly after cyst rupture, fatal Eosinophils are uncommon. In the reported case, the patient's symptoms were fairly mild. There was no allergic reaction nor any severe pain and only slight bleeding. Hydatid disease would not be the first disease to consider with this mild and fairly common presentation.

Calcification, which usually requires 5-10 years for development, occurs quite commonly with hepatic cysts but rarely with pulmonary cysts, and bone hydatid cysts do not undergo calcification. Total calcification of the cyst wall suggests that the cyst may be nonviable (4).

Albendazole is preferred because it has better bioavailability. It is given at a dosage of $10-15 \mathrm{mg} / \mathrm{kg}$ body weight per day in two divided doses, and the usual dose is $800 \mathrm{mg}$ daily. A newer benzimidazole compound, oxfendazole, has been studied and preliminary results suggest it may be a more effective compound.

\section{CONCLUSION}

In conclusion, hydatid disease presents with a variety of symptoms and sometimes with special imaging features. Because it is endemic in Greece, this disease should always be included in the differential diagnosis of hemoptysis and fever, especially in patients who live in agricultural areas. Diagnosis is obtained by imaging evaluation (Chest X-ray or CT scan), supported by serology in the majority of cases (5).

\section{REFERENCES}

1. Stamatakos M, Sargedi C, Stefanaki Ch, Safioleas C, Matthaiopoulou I, Safioleas M. Anthelminthic treatment: an adjuvant therapeutic strategy against Echinococcus granulosus. Parasitol Int. 2009 Jun; 58(2):115-20. doi: 10.1016/j.parint.2009. 01.002. Epub 2009 Jan 20.

2. Gavidia CM, Gonzalez AE, Lopera L, Jayashi C, Angelats R, Barron EA, et al. Evaluation of nitazoxanide and oxfendazole efficacy against cystic echinococcosis in naturally infected sheep. Am J Trop Med Hyg. 2009 Mar;80(3):367-72

3. Bygott JM, Chiodini PL. Praziquantel: neglected drug? Ineffective treatment? Or therapeutic choice in cystic hydatid disease? Acta Trop. 2009 Aug;111(2):95-101. doi: 10.1016/j.actatropica.2009.04.006. Epub 2009 Apr 16.

4. Haralabidis S, Diakou A, Frydas S, Papadopoulos E, Mylonas A, Patsias $A$, et al. Long-term evaluation of patients with hydatidosis treated with albendazole and praziquantel. Int $\mathrm{J}$ Immunopathol Pharmacol. 2008 Apr-Jun;21(2):429-35.

5. Moro P, Schantz PM. Echinoccosis: a review. Int J Infect Dis. 2009 Mar;13(2):125-33. doi: 10.1016/j.jijid.2008.03.037. Epub 2008 0ct 19. 